\title{
CRIAÇÃO DE VALOR NO PROCESSO DE DESENVOLVIMENTO DE PRODUTOS: UMA AVALIAÇÃO DA APLICABILIDADE DOS PRINCÍPIOS E PRÁTICAS ENXUTAS
}

\section{VALUE CREATION IN THE PRODUCT DEVELOPMENT PROCESS: AN ASSESSMENT OF THE LEAN PRINCIPLES AND PRATICES APLICABILITY}

\author{
Marcio Cardoso Machado ${ }^{1}$; Nilton Nunes Toledo ${ }^{2}$ \\ ${ }^{1}$ Escola Politécnica da Universidade de São Paulo- EPUSP - São Paulo - Brasil \\ marcio.cardoso@poli.usp.br \\ ${ }^{2}$ Escola Politécnica da Universidade de São Paulo-EPUSP - São Paulo - Brasil tolenil@usp.br
}

\begin{abstract}
Resumo
Em um ambiente intenso e dinâmico, o desenvolvimento de novos produtos e processos crescentemente tem se tornado o principal foco de competição, a qual está sendo dirigida elas seguintes forças: a intensa competição internacional; mercados segmentados com consumidores sofisticados; e as variadas transformações tecnológicas. Portanto, o resultado de um processo de desenvolvimento de produto deve ser um conjunto de definições que permita realização do produto e, simultaneamente, crie o valor desejado para os stakeholders. Desta forma, a possibilidade de implementação de princípios enxutos no Processo de Desenvolvimento de Produtos(PDP) aparece como uma oportunidade de estudo ainda pouco explorada. O presente trabalho apresenta os resultados da análise da aplicabilidade de princípios e práticas enxutas no PDP, os quais foram obtidos a partir de dois estudos de casos realizados em empresas responsáveis pelo desenvolvimento de itens para a indústria aeronáutica. Para tanto, foram feitas visitas quinzenais ao longo de oito meses, e durante as visitas foram utilizados diferentes instrumentos de coleta de dados: entrevistas semi-estruturadas com a alta-administração, gerentes de processos e pessoal técnico, cujo objetivo foi identificar de que forma o PDP é compreendido pelos distintos níveis hierárquicos, e a análise de documentos relativos ao desenvolvimento de produtos passados $e$ atuais. Para verificar o alinhamento dos processos atuais com os princípios e práticas enxutas foram utilizados formulários elaborados com base na literatura específica, e refinados através da prática industrial observada. Também são discutidas as possíveis implicações para a teoria e prática gerencial, assim como, são oferecidas sugestões para futuras pesquisas.
\end{abstract}

Palavras-chave: valor, desenvolvimento de produtos, princípios enxutos.

\section{Introdução}

Em um ambiente intenso e dinâmico, o desenvolvimento de novos produtos e processos crescentemente tem se tornado o principal foco de competição (Wheelwright \& Clark, 1992). Esta 
nova competição industrial fortemente focada no desenvolvimento de produto está sendo dirigida por forças que têm surgido, nas ultimas décadas, em muitas indústrias ao redor do mundo. São elas: o aparecimento de uma intensa competição internacional; a criação de mercados segmentados com consumidores sofisticados; e as variadas transformações tecnológicas (Clark \& Fujimoto, 1991).

Examinando a literatura sobre inovação industrial, verifica-se que os produtos aeronáuticos tornaram-se “dominante design” (Utterback, 1996), figura 1. e entraram em uma fase específica no seu ciclo de vida. A inovação, nesta fase, está centrada na melhoria incremental do produto, especialmente no que diz respeito à produtividade; à qualidade; à tecnologia de processo e às tecnologias que oferecem produtos substitutos superiores (Murman, 2000) figura 2.

Figura 1: A Dinâmica da Inovação

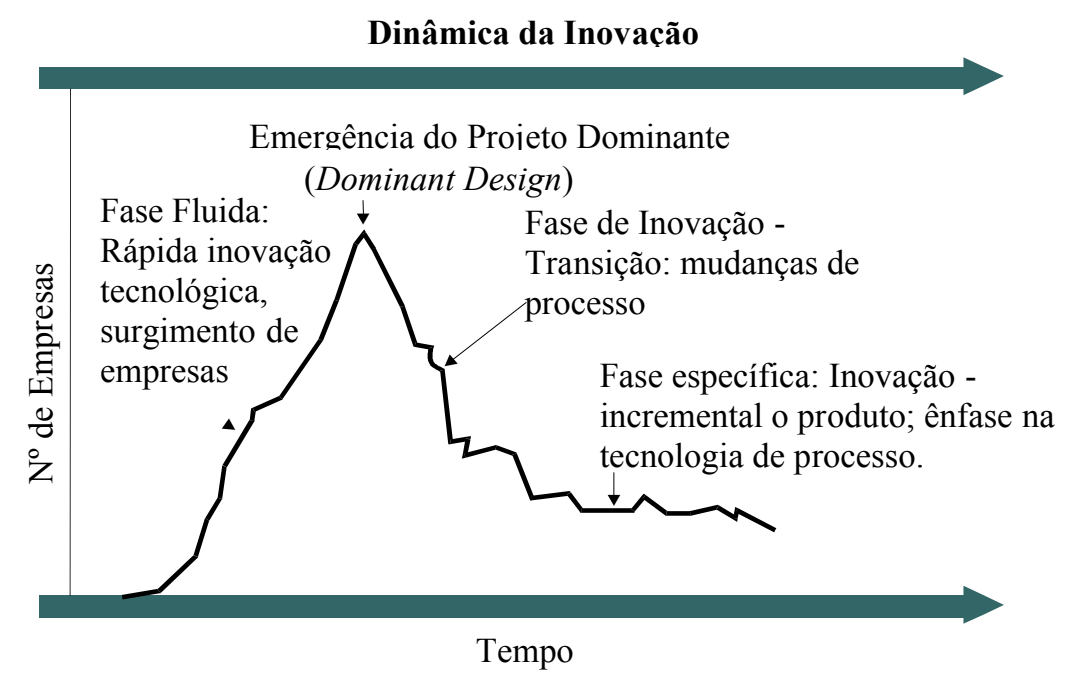

Fonte: Utterback (1996).

Figura 2: A Dinâmica da Inovação em três setores específicos

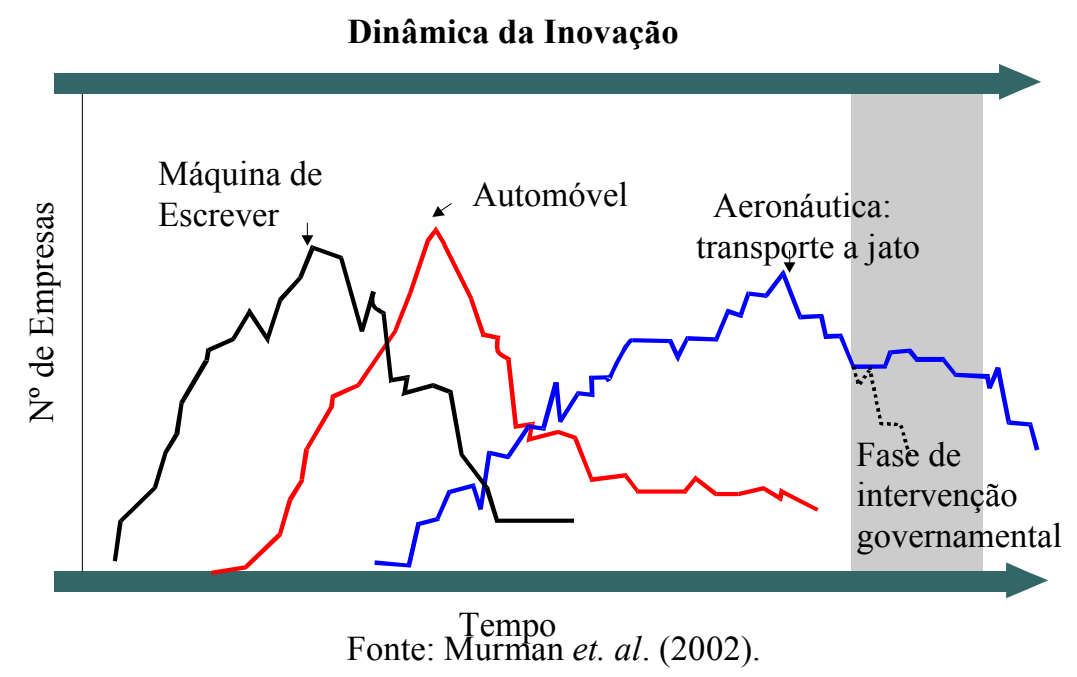


A possibilidade de implementação de princípios enxutos no processo de desenvolvimento de produtos aparece como uma oportunidade de estudo, visto que, segundo Krishnan \& Ulrich (2001), os benefícios de novas ferramentas para gerenciar o conhecimento acerca do produto e para dar suporte à tomada de decisão no desenvolvimento necessitam ser explorados em grandes detalhes.

O primeiro princípio enxuto é a especificação de valor. Contudo, durante o processo de desenvolvimento de produtos o valor é difícil de ser entendido. A complexidade do processo, a distância do consumidor final, a alternância das condições de mercado, o aparecimento de novas tecnologias e as incertezas de performance técnica, custo e programação, tornam a definição de valor baseada nas necessidades dos clientes uma tarefa de difícil execução.

Trabalhos anteriores como Stanke (2001) e Chase (2001) oferecem modelos para a representação destas complexidades.

\section{Inovação}

A inovação tecnológica tem sido, através da história, a força propulsora para o desenvolvimento industrial, para o crescimento da produtividade e, de fato, para a elevação de nosso padrão de vida. Porém, estudos mais aprofundados sobre seu papel e influência na indústria são um fenômeno relativamente recente (Abernathy \& Clark, 1985).

Para Utterback (1996), apesar do aumento do interesse pela influência de novas tecnologias na estrutura da indústria e na concorrência de mercado, poucos estudantes pesquisadores têm examinado relações entre processos de produção, melhoria do processo, avanços de produtividade e competitividade.

Abernathy \& clark (1985) já ressaltavam que alguns trabalhos têm examinado as determinantes da inovação somente com foco na demanda e na estrutura de mercado, e o papel efetivo da inovação não estava sendo discutido. Desta forma, apenas os aspectos da indústria ou da empresa que estimulam ou retardam o avanço tecnológico eram o centro das investigações.

A inovação não é um fenômeno que pode ser unificado, pois algumas inovações rompem, destroem e tornam obsoletas tecnologias estabelecidas, enquanto outras as refinam e as melhoram, assim como seus efeitos nos sistemas de produção podem ser diferentes daqueles que ocorrem nas interações entre consumidores e mercado. Estas diferenças ressaltam que a significância competitiva de uma inovação depende do que ela representa para o valor e para a aplicabilidade da competência estabelecida.

A criação de nichos de mercado e o estabelecimento de uma nova arquitetura de produto envolvem um tipo de inovação que é visível e aparentemente lógica. No sentido oposto, existe um tipo de inovação que normalmente não é visível. Esta, porém, possui um importante efeito 
cumulativo no custo e performance do produto. Abernathy \& Clark (1985) chamam-na de inovação "Regular" e faz parte do que consta da estrutura do modelo que os autores intitularam "Mapa de Transiliência". Neste tipo de inovação, as mudanças ocorrem nas competências técnicas e de produção estabelecidas e, por se tratar de uma mudança incremental na tecnologia de processo, tende a elevar a produtividade e aumentar a capacidade do processo.

\section{Os Princípios Enxutos no Ambiente de Desenvolvimento de Produtos}

Para Womack \& Jones (2003), a aplicação dos cinco princípios enxutos nos processos e em toda a empresa conduzirá ao que eles chamam de estado "enxuto". Este estado enxuto é resultante da eliminação de desperdícios nas operações, de tal forma que os produtos possam ser desenvolvidos com uma mínima parcela dos custos totais de material, tempo e esforço humano.

Estes princípios são:

- Princípio do Valor: especificar de forma precisa o valor;

- Princípio do Fluxo do Valor: identificar o fluxo do valor;

- Princípio do Fluxo: fazer com que o valor identificado flua;

- Princípio do Sistema Puxado: deixar que o consumidor puxe o valor; e

- Princípio da Perfeição: esforço para a perfeição.

A tabela 1 compara a aplicação dos princípios enxutos no ambiente de manufatura e no desenvolvimento de produtos.

Tabela 1 - Comparação entre a aplicação dos princípios enxutos no ambiente de manufatura e no desenvolvimento de produtos.

\begin{tabular}{l|l|l}
\hline & \multicolumn{1}{|c}{ Manufatura } & \multicolumn{1}{|c}{ Desenvolvimento de Produtos } \\
\hline Definir valor & $\begin{array}{l}\text { Visível em cada etapa, meta } \\
\text { definida. }\end{array}$ & Difícil de enxergar, metas emergentes. \\
\hline $\begin{array}{l}\text { Identificar o fluxo } \\
\text { do valor }\end{array}$ & Partes e material. & Informação e conhecimento. \\
\hline $\begin{array}{l}\text { Fazer o processo } \\
\text { fluir }\end{array}$ & As interações são desperdícios. & $\begin{array}{l}\text { As interações freqüentemente são } \\
\text { benéficas. }\end{array}$ \\
\hline Sistema Puxado & Dirigido pelo tempo takt & Dirigido pelas necessidades da empresa. \\
\hline Perfeição & Processo sem erros e repetível. & $\begin{array}{l}\text { Processo permite a inovação e reduz } \\
\text { tempo de ciclo- }\end{array}$ \\
\hline
\end{tabular}

Um número significativo dos exemplos de aplicação e discussão dos princípios enxutos tem sido amplamente focado na área da manufatura, principalmente no que tange ao fluxo de valor. Segundo Slack (1998), existem exemplos na indústria onde estes princípios conseguiram transformar com sucesso empresas de manufatura. Porém, os processos utilizados na manufatura e 
no processo de desenvolvimento de produtos são diferentes, assim como existem diferenças no produto que estes processos produzem.

A habilidade de colocar no mercado produtos inovadores e de alta qualidade tem rapidamente se tornado fato condicionante para o sucesso de uma empresa. $\mathrm{Na}$ indústria automobilística, isto é particularmente verdadeiro, pois a drástica redução no tempo de ciclo de desenvolvimento e o aumento significativo da qualidade dos produtos têm sido fatores cruciais para a distinção entre as melhores empresas e o resto da indústria (Sobeck, Ward and Liker 1999).

Qual a relação disso com a mentalidade enxuta? A velocidade da colocação de um produto no mercado é alcançada tanto através da maximização da eficiência no projeto do produto como através dos estágios de manufatura. A pesquisa comparativa realizada por Morgan (2002) na indústria automobilística sugere que o desempenho no desenvolvimento de produtos pode ser beneficiado pelas mesmas ferramentas e métodos enxutos que dirigiram o renascimento da manufatura norte-americana.

Para Morgan (2002), o sistema Toyota de desenvolvimento de produto pode oferecer, à engenharia, a mesma transformação inovadora que o Sistema de Produção Toyota ofereceu à manufatura. O sistema Toyota de desenvolvimento de produto facilita a colocação rápida no mercado de produtos com qualidade superior e através de uma manufatura ágil e eficiente.

Em resumo, a Toyota tem conseguido, sistematicamente, desenvolver produtos e respectivos processos com menores custos, mais rapidamente e com melhor qualidade do que seus concorrentes. E este sucesso, em grande medida, deve-se à ampliação da aplicação da filosofia implícita no Sistema Toyota de Produção para as mais diversas funções empresariais.

O processo de desenvolvimento dos produtos, além de ser capaz de captar as dimensões do que seria valor para o cliente, é orientado pelas implicações operacionais e tecnológicas que estarão, num momento seguinte, presentes nos processos de manufatura, dentro e fora da empresa.

Assim como, por anos, os reais conceitos e práticas da Toyota na manufatura ficaram escondidos ou pouco compreendidos, agora o desafio é desvendar a "caixa preta" presente nos conceitos da empresa nesta dimensão dos negócios.

O problema a ser investigado por este trabalho é se os princípios enxutos, que têm facilitado, na manufatura, a transição para um estado enxuto, podem ser utilizados de forma efetiva na melhoria do processo de desenvolvimento de produtos.

\section{Estudos de Campo}

As empresas estudadas foram escolhidas em função do estágio de inovação praticado pela indústria da qual fazem parte. A indústria aeronáutica, como já mencionado neste trabalho, encontra-se na fase específica, definida por Utterback (1996), e portanto necessita buscar inovações 
relacionadas com processos, visto que seus produtos já alcançaram o que podemos chamar de dominant design. Os estudos realizados objetivaram avaliar o grau de alinhamento das empresas com os princípios e práticas enxutas. Para tanto, foi utilizado um formulário híbrido adaptado do Lean Self Assessment (LESAT) proposto pelo MIT. Com este instrumento de pesquisa foi possível coletar informações através dos seguintes métodos: entrevistas, observação direta e pesquisa documental, além de permitir a mensuração do grau de alinhamento das empresas com os princípios enxutos. Para efeito deste artigo somente serão apresentados os resultados relacionados com grau de alinhamento. A empresa "A" tem sido reconhecida pelo excelente desempenho no desenvolvimento de produtos e na integração de sistemas. A empresa " $\mathrm{B}$ " tem um volume grande de projetos de desenvolvimento de itens para a aviação militar e para equipamento de apoio ao solo.

\subsection{Descrição das Empresas}

\subsubsection{Empresa A}

A empresa A pertence à indústria aeronáutica brasileira, porém a sua atuação se expande para além das fronteiras do território nacional. Seus principais produtos são aeronaves de transporte regional de passageiros e aviões de emprego militar, alem de atuar como fornecedora de partes para outras empresas da indústria aeronáutica internacional. Em função de alguns critérios de confidencialidade as informações dos questionários foram obtidas basicamente através de três fontes: a partir de entrevistas realizadas durante três visitas à fábrica, visitas estas que ocorreram ao longo de seis meses; observação direta durante as mesmas visitas e artigos e trabalhos científicos sobre a empresa em questão.

\subsubsection{Empresa B}

A empresa B é uma empresa governamental cuja principal atividade é o desenvolvimento de itens aeronáuticos de reposição para a frota de aviões da Força Aérea Brasileira. O processo de desenvolvimento de produtos nesta empresa tem como principais características: um alto grau de simultaneidade de produtos sendo desenvolvidos e também uma quase-totalidade de desenvolvimento puxado a partir da necessidade dos clientes. Para o preenchimento dos questionários, foram realizadas visitas mensais ao longo de um ano. Os entrevistados pertenciam à alta administração, ao corpo de engenheiros e ao quadro técnico. 


\subsection{Método Utilizado}

Para avaliar o grau de alinhamento das empresas com os princípios enxutos, foram realizados dois estudos de caso. No instrumento de coleta de dados, existiam seis questionamentos acerca das práticas enxutas, que podem estar relacionados com um ou mais princípios enxutos. Cabe ressaltar que, segundo Slack (1998), o princípio do sistema puxado não se mostrou aplicável no contexto de desenvolvimento de produtos, a não ser em situações específicas onde o processo de desenvolvimento de produtos é desencadeado pelo próprio cliente. Sabendo que nenhuma das empresas analisadas tem um processo de desenvolvimento enxuto formalizado, não se pretendeu com esta avaliação verificar o grau de maturidade do processo, somente o grau de alinhamento.

Os seis questionamentos encontram-se descritos abaixo:

- Q1 - O processo de desenvolvimento de produto é formalizado e compreendido? (princípio do fluxo do valor).

- Q2 - Os clientes e demais stakeholders (acionistas, funcionários, etc.) são regularmente envolvidos no processo de desenvolvimento? (princípio do valor).

- Q3 - As preocupações iniciais dos stakeholders acerca do projeto e do desenvolvimento são consideradas e incorporadas no processo tão cedo quanto possível? (princípio do valor).

- Q4 - As interações desnecessárias no ciclo de desenvolvimento são removidas? (princípio do fluxo).

- Q5 - O ciclo de desenvolvimento tem sido simplificado e alinhado ao caminho crítico? (princípio do fluxo e princípio da perfeição).

- Q6 - Produtos e processos são desenvolvidos de forma simultânea? (princípio do fluxo e princípio da perfeição).

Para cada questionamento foi utilizada uma escala de pontuação que variou de 1 a 5 . Os valores mais próximos de 1 representavam pouco alinhamento com a prática enxuta avaliada, por conseqüência os valores mais próximos de 5 representavam alto grau de alinhamento com as práticas enxutas.

\subsection{Resultados Obtidos}

As respostas das empresa estudas revelam importantes diferenças, no que diz respeito ao processo de desenvolvimento de produtos. Os resultados obtidos foram representados de forma gráfica para uma melhor visualização dos resultados obtidos (figuras 3 e 4). 
O primeiro ponto que se deve ressaltar acerca das diferenças de desenvolvimento de produtos entre as empresas estudadas está relacionado com a formalização do processo (Q1). Ambas as empresas estudadas possuem um processo formal de desenvolvimento de produto, porém, em função da complexidade, existe um dificuldade maior para a empresa "A" em fazer com que todos os participantes o compreendam. Na empresa "B", cujo número de envolvidos é menor verificou-se uma melhor compreensão acerca do processo. Os esforços de melhoria do processo se mostraram mais evidentes na empresa "A".

Figura 3 - Grau de alinhamento da empresa "A".

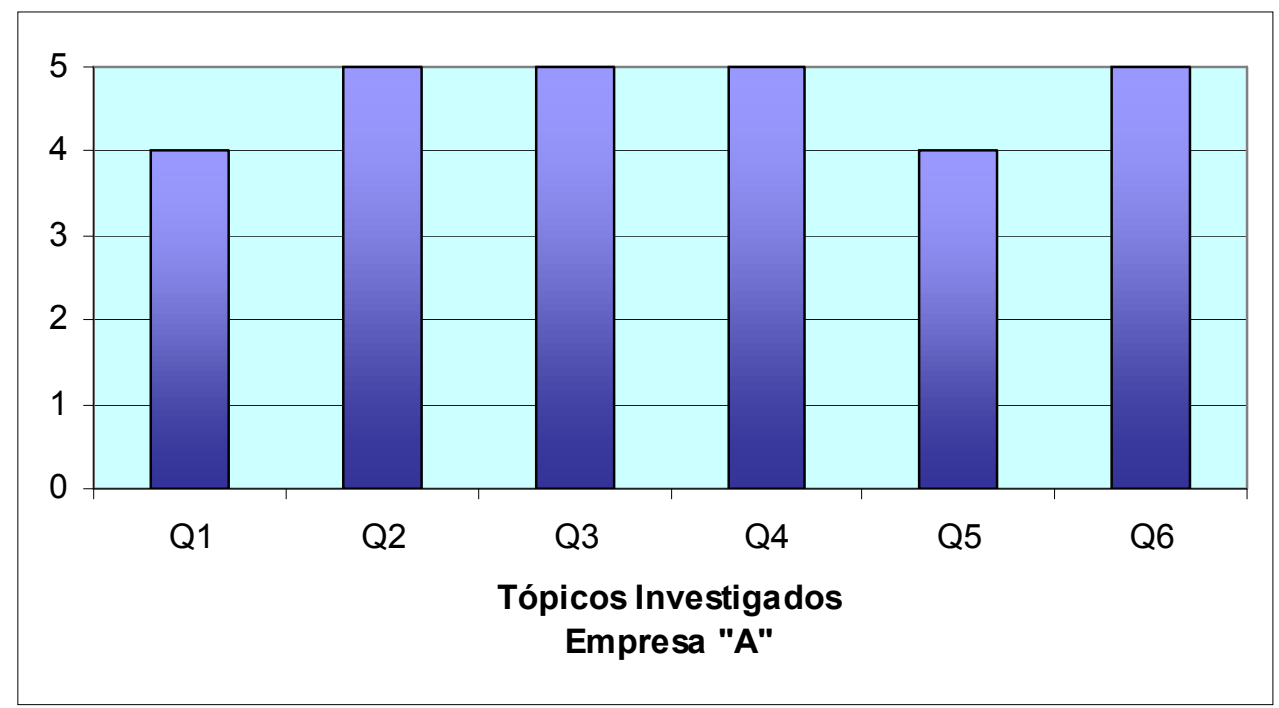

No segundo e terceiro ponto (Q2 e Q3) - envolvimento dos stakeholders - observou-se que, na empresa "A", este envolvimento acontece ao longo de todo o processo, com mais ênfase em fases específicas. Enquanto na empresa "B" o envolvimento se dá somente no início do processo e de forma parcial.

$\mathrm{Na}$ quarto questionamento do formulário (Q4) - fluxo de informações - foram obtidas as seguintes informações. A empresa "A" possui um software que disponibiliza as informações relativas ao processo de desenvolvimento e ao mesmo tempo oferece a possibilidade de avaliação do impacto que as atividades a jusante podem ter nas atividades a montante. Na empresa "B", não foi identificada qualquer atividade de avaliação dinâmica do fluxo de informações. 
Figura 4 - Grau de alinhamento da empresa "B".

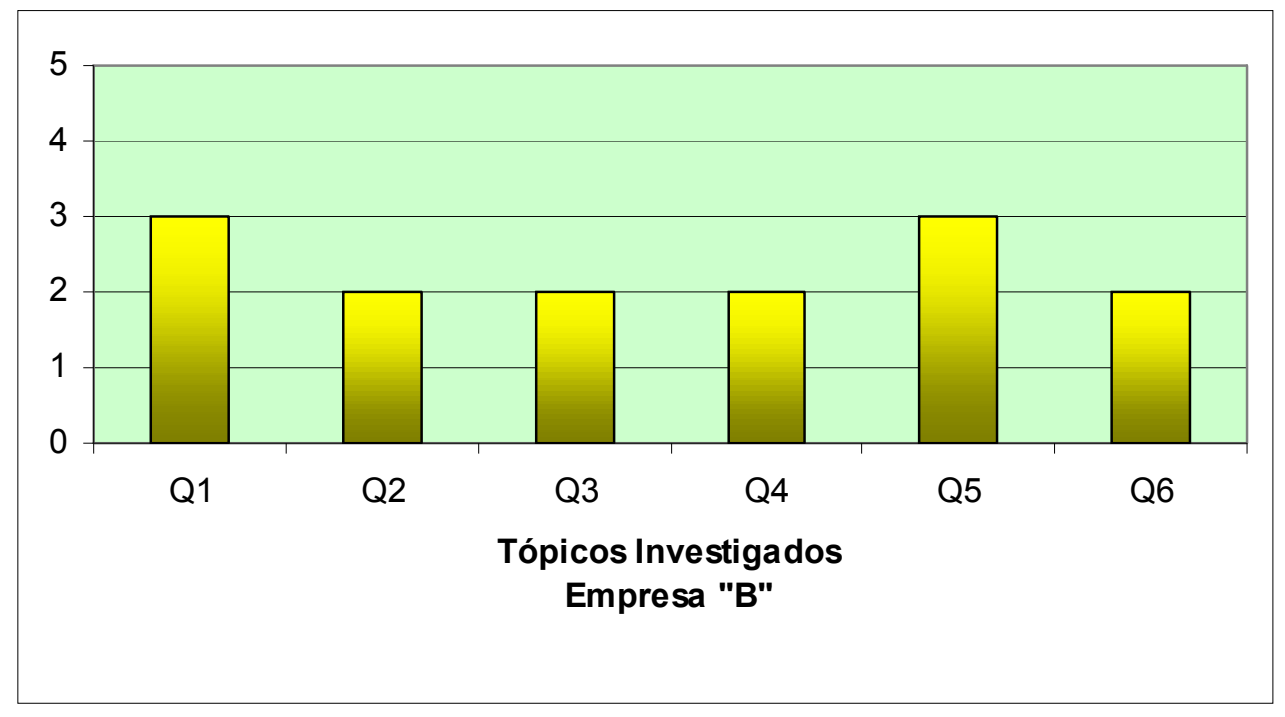

O quinto questionamento (Q5) tratou da simplificação do processo. Para a empresa “A”, este objetivo é claro e várias iniciativas são tomadas neste sentido, como por exemplo: a utilização conjunta de ferramentas de CAD, CAM, CAE e CIM, e o uso de mock up eletrônico. Na empresa “B”, não se observou ações de melhoria no sentido de simplificar o processo de desenvolvimento.

Com relação a simultaneidade no desenvolvimento de produtos e processos (Q6), verificouse que a empresa "A" utiliza times integrados de desenvolvimento, com envolvimento de stakeholders, além da utilização de métricas de avaliação. Na empresa "B", a utilização de times ocorre somente em um momento específico a montante do processo.

\subsection{Considerações}

A análise dos casos revelou, primeiramente, que se trata de empresas com grandes diferenças nos mais variados aspectos, tanto no que se refere ao processo de desenvolvimento de produtos quanto nas questões organizacionais e tecnológicas. Os produtos desenvolvidos também são consideravelmente diferentes. Em segundo lugar, revelou que as comunalidades existentes entre as empresa dizem respeito ao tipo de indústria e à confiabilidade exigida de seus produtos.

A partir a aplicação dos formulários de pesquisa e com base nas informações obtidas foi possível avaliar o alinhamento das empresas com relação aos princípios enxutos. A empresa " $\mathrm{A}$ " tem, em várias etapas do processo de desenvolvimento, um alto grau de alinhamento.

Esta convergência leva ao entendimento de que, mesmo sem uma abordagem estruturada para implementação dos princípios enxutos no processo de desenvolvimento de produtos, a empresa caminha na direção de um processo enxuto de desenvolvimento. 
A empresa $\mathrm{B}$, através da avaliação do questionário, não apresentou alinhamento com os princípios enxutos. E em face dos problemas identificados, não só no processo de desenvolvimento mas também nos produtos que se originam deste processo, é pertinente inferir que as práticas identificadas na empresa $\mathrm{A}$, a despeito das diferenças observadas entre as empresas, podem ser úteis à empresa B.

As respostas das empresas estudadas também revelaram importantes diferenças, no que diz respeito ao processo de desenvolvimento de produtos e conseqüentemente no grau de alinhamento destes processos com os princípios. A empresa "A", com maior alinhamento, é também a que tem sido reconhecida pela excelência em desenvolvimento de projetos e integração de sistemas. Isto reforça a ideia de Morgan (2002) de que os princípios e conceitos enxutos presentes no processo de desenvolvimento de produtos, então utilizados pela Toyota, podem ser úteis a outras empresas mesmo que não pertencentes à indústria automobilística.

Na empresa "B", o grau de alinhamento foi considerado baixo, assim como foi observado que existem vários pontos de ineficiência no que tange à melhoria do processo. Alguns indicadores, não detalhados no copo deste trabalho mas observados durante as visitas realizadas ao longo de um ano na empresa "B", revelaram que a empresa tem enfrentado problemas de retrabalho em função de interações tardias.

Portanto, a inovação do processo de desenvolvimento de produtos através da utilização de princípios enxutos foi identificada como uma possibilidade factível. Este breve trabalho não pretende esgotar a discussão sobre o tema. Ao contrário, pretende oferecer um marco inicial para futuras pesquisas, no cenário nacional, relacionadas com a utilização de princípios enxutos no processo de desenvolvimento de produtos.

\footnotetext{
Abstract

In an intense and dynamic environment, the development of new products and processes increasingly has been become the main focus of competition, which is being directed by the following forces: the intense international competition; markets segmented with sophisticated consumers; and the varied technological transformations. Therefore, the result of a process of product development must be a set of definitions that allows the accomplishment of the product and, simultaneously, creates the value desired for stakeholders. In such a way, the possibility of implementation of lean principles in the Product Process Development (PPD) appears as a study opportunity area still little explored. The present work presents the results of the analysis of lean principles and practices applicability in the PPD, which had been gotten from two case studies carried through companies that develop parts for the aeronautical industry. In such a way, biweekly visits throughout eight months had been made, and during the visits different instruments of data collection had been used: semi-structuralized interviews with the directors, engineers, processes managers and technician staff, whose objective was to identify how PPD is understood by the distinct hierarchic levels, and to analyze documents of the last and current product developments. To verify the alignment of the current processes with the lean principles and practices it was used forms elaborated on the basis of specific literature, and refined through the industrial practices
} 
observed. Also are argued the possible implications for the managerial theory and practice, as well as, are offered suggestions future research.

Key-words: value, product development, lean principles.

\section{Referências}

ABERNATHY W.J. e CLARK, K.B. Innovation: mapping the winds of creative destruction. Research Policy 14, pag. 3-32. Elsevier Science Publishers B. V. North-Holland: 1985.

CHASE, James P. Value Creation in the Product Development Process. Thesis (master in science). Massachusetts Institute of Technology. Cambridge: 2001.

CLARK, K. FIJIMOTO, C. Product Development Performance: strategy, organization and management in the world auto industry. HBS Press: 1991.

KRISHNAN, V. ULRICH, Karl T. Product Development Decisions: a review of the literature. Management Science. V.47, n.1, p.1-21: 2001.

MORGAN, James M. High performance product development: a systems approach to a lean product development process. Thesis (Phd) in industrial and operations engineering. The University of Michigan: 2002.

MURMAN, E. at. al. Value in Aerospace Industry. Palgrave: New York, 2002.

SLACK, R. A. The application of lean principles to the military aerospace product development process. Thesis (Master in Science). Massachusetts Institute of Technology: Cambridge, 1998.

SOBEK II, R.P.; WARD, A.C.; LIKAR, J.K. Toyota's principles of set-based concurrent engineering. Sloan Management Review, Cambridge, v. 40, p 67-83, winter 1999.

STANKE, A. A framework for achieving lifecycle value in product development. Thesis (Master in Science). Massachusetts Institute of Technology: Cambridge, 2001.

UTTERBACK, James M. Suáres, Fernando F. Innovation, competition, and industry structure. Research Policy 22 , pág 1-22. North-Holland: 1993.

WARD, A. CRISTIANO. SOBEK. The Second Toyota Paradox: how delaying decisions can make better car faster. Sloan Management Review - 1995.

WHEELWRIGHT \& CLARK. Revolutionizing product development: quantum leaps in speed, efficiency and quality. 1992.

WOMACK, J. Jones, D. Lean Thinking: banish waste and create wealth in your corporation. Free Press: New York, 2003.

\section{Dados completos de todos os autores:}

\section{Nome completo: Marcio Cardoso Machado}

Filiação institucional: Escola Politécnica da USP

Departamento: Engenharia de Produção

Função ou cargo ocupado: Doutorando

Endereço completo para correspondência (bairro, cidade, estado, país e CEP): Pç Campo de Bagatelle, 235. B1-03 Apto-202, Santana, São Paulo, Brasil. CEP 02012-000. 
Telefones para contato: (011) 6221-1969, (011) 8316-4545

e-mail: marcio.cardoso@poli.usp.br

\section{Nome completo: Nilton Nunes Toledo}

Filiação institucional: Escola Politécnica da USP

Departamento: Engenharia de Produção

Função ou cargo ocupado: Professor

Endereço completo para correspondência (bairro, cidade, estado, país e CEP): Rua Belmonte, 269.

Alto da Lapa, São Paulo, Brasil. CEP 05088-050.

Telefones para contato: (011) 3835-2862, (011) 9121-2174

e-mail: tolenil@usp.br

Recebido para publicação em: 08/08/06

Aceito para publicação em: 12/09/06 